\title{
Progressive dyspnoea in a patient with idiopathic non-cirrhotic portal hypertension
}

\author{
Sarah Cullivan $\mathbb{1}^{\text {, }}$, Brian McCullagh and Sean Gaine \\ National Pulmonary Hypertension Unit, Mater Misericordiae University Hospital, Dublin, Ireland.
}

Corresponding author: Sarah Cullivan (sarah.cullivan@ucdconnect.ie)

Shareable abstract (@ERSpublications)

This case of progressive dyspnoea in a 43-year-old with idiopathic non-cirrhotic portal hypertension highlights important pulmonary vascular complications of chronic liver disease https://bit.ly/3rwEkhP

Cite this article as: Cullivan S, McCullagh B, Gaine S. Progressive dyspnoea in a patient with idiopathic non-cirrhotic portal hypertension. Breathe 2022; 18: 210168 [DOI: 10.1183/20734735.0168-2021].

Copyright @ERS 2022

Breathe articles are open access and distributed under the terms of the Creative Commons Attribution Non-Commercial Licence 4.0.

Received: 12 Nov 2021

Accepted: 12 Jan 2022
A 43-year-old gentleman was referred to a pulmonologist for further assessment of a 3-year history of progressive dyspnoea on exertion. His background was significant for idiopathic non-cirrhotic portal hypertension, for which he underwent splenectomy and portosystemic shunt formation as a child to manage complications related to hypersplenism. His medical history was also notable for mild kyphoscoliosis. He was a lifelong nonsmoker and denied alcohol consumption or illicit drug use.

He reported New York Heart Association functional class II dyspnoea and denied other cardiorespiratory symptoms including chest pain, orthopnoea, limb oedema or syncope. Examination revealed normal resting saturations of $98 \%$ on room air in the standing and supine positions. There was grade 1 digital clubbing and normal vesicular breath sounds on auscultation. Cardiovascular examination revealed a right ventricular heave, a loud pulmonary component of the second heart sound (P2) and he was euvolemic.

In light of these signs an echocardiogram with right heart protocol was requested.

\section{Task 1}

Which feature(s) on echocardiography is/are suggestive of pulmonary hypertension (PH), as per the 2015 European Society of Cardiology/European Respiratory Society guidelines for the diagnosis and treatment of PH in a symptomatic patient?
a) Flattening of the intraventricular septum
b) Peak tricuspid regurgitation velocity $>3.4 \mathrm{~m} \cdot \mathrm{s}^{-1}$
c) Pulmonary artery diameter $>10 \mathrm{~mm}$
d) Right ventricle/left ventricle basal diameter ratio $>1.0$
e) Peak tricuspid regurgitation velocity $\leqslant 2.8 \mathrm{~m} \cdot \mathrm{s}^{-1}$

Go to Answers >>

Echocardiography demonstrated a dilated right atrium and right ventricle, a pulmonary artery diameter of $34 \mathrm{~mm}$ and a peak tricuspid regurgitation velocity of $3.85 \mathrm{~m} \cdot \mathrm{s}^{-1}$. The left heart was normal in size and function.

These echocardiographic features suggested a high pretest probability of $\mathrm{PH}$ and a right heart catheterisation was requested to assess this further (table 1).

The right heart catheterisation revealed a precapillary pattern of $\mathrm{PH}$ (table 1). Potential aetiologies of $\mathrm{PH}$ were explored. Investigations including autoimmune and hepatitis serology, spirometry, ventilation/ perfusion $\left(V^{\prime} / Q\right)$ imaging and computed tomography chest were all normal. Hepatic synthetic function was 
Table 1 Right heart catheterisation revealed a precapillary pattern of $\mathrm{PH}$ with $\mathrm{mPAP}>20 \mathrm{mmHg}$, PAWP $<15 \mathrm{mmHg}$ and PVR $>3$ WU

Mean pulmonary artery pressure (mPAP)

$46 \mathrm{mmHg}$

Pulmonary vascular resistance (PVR)

Pulmonary artery wedge pressure (PAWP)

6.25 Wood Units (WU)

Mean right atrial pressure

Cardiac output

Pulmonary artery saturations

$11 \mathrm{mmHg}$

$6 \mathrm{mmHg}$

$5.6 \mathrm{~L} \cdot \mathrm{min}^{-1}$

$74 \%$

normal and there was no evidence of cirrhosis on liver imaging. Doppler ultrasonography demonstrated portal hypertension, which was consistent with the patient's known history of same.

Task 2

Given the above findings and in the context of established portal hypertension what is the most likely aetiology of $\mathrm{PH}$ in this case?

a) Hepatopulmonary syndrome

b) Chronic respiratory failure due to kyphoscoliosis

c) Chronic thromboembolic pulmonary hypertension

d) Portopulmonary hypertension

e) Left heart disease

Go to Answers >>

The patient was diagnosed with PoPH due to idiopathic non-cirrhotic portal hypertension.

Task 3

Which of the following statements regarding PoPH is true? (One correct answer)

a) PoPH only occurs in patients with advanced cirrhosis

b) The prescription of a $\beta$-blocker for prophylaxis of variceal bleeding in these cases may be associated with deleterious effects on exercise capacity and pulmonary haemodynamics

c) $\mathrm{PoPH}$ is an absolute contraindication to liver transplantation

d) Endothelin receptor antagonists are contraindicated due to potential liver toxicity

Go to Answers >>

The patient commenced double combination therapy with a phosphodiesterase type 5 inhibitor and an ERA and he remains well 7 years following this diagnosis.

\section{Discussion}

This case of PoPH in a 43-year-old gentleman highlights important pulmonary vascular complications of chronic liver disease.

PoPH is defined as PAH associated with portal hypertension and is allocated to group one PAH [9, 10]. It can develop in patients with portal hypertension with or without concomitant liver disease, such as in this case of non-cirrhotic portal hypertension [3]. The histopathology is indistinguishable from other PAH subgroups and is characterised by intimal proliferation, smooth muscle hypertrophy and plexiform lesions $[9,11]$. However, the epidemiology and pathobiology are incompletely defined [9]. The reported incidence and prevalence of $\mathrm{PoPH}$ are heterogeneous, and registry data indicate that it may account for $5 \%$ to $17.6 \%$ of PAH cases $[6,12]$. Reduced hepatic clearance of vasoactive mediators, pathogens and toxins have been implicated in the pathobiology of PoPH [9]. Increased shear stress due to augmented blood flow through the pulmonary vasculature associated with high cardiac output may also play a role [9].

Right heart catheterisation is required to diagnose $\mathrm{PAH}$ and demonstrate a mPAP >20 mmHg, PAWP $<15$ mmHg and PVR >3 WU [13]. While the severity of PoPH may be graded by mPAP values (table 2), it is important to consider additional haemodynamic parameters and right heart function. Invasive measurement of hepatic venous pressure gradient $(>5 \mathrm{mmHg})$ was considered the gold standard to diagnose portal hypertension, however, noninvasive techniques such as doppler ultrasonography are increasingly employed [14]. Furthermore, it should be noted that the hepatic venous pressure gradient may be normal in patients with extrahepatic portal hypertension. 
Table 2 Key features of PoPH and hepatopulmonary syndrome (HPS)

PoPH

\begin{tabular}{l}
\hline Portal hypertension \\
Chronic liver disease \\
\hline PVR \\
\hline Diagnostic characteristic \\
\hline
\end{tabular}

Grading of severity

Grading of severity

Treatment
Always present

Sometimes present

High

Demonstration of PAH: MPAP

$>20$ mmHg; PVR >3 WU; PAWP

$<15 \mathrm{mmHg}$

Right heart catheterisation

mPAP:

Mild: $25-34 \mathrm{mmHg}$

Moderate: $35-44 \mathrm{mmHg}$

Severe: $\geqslant 45 \mathrm{mmHg}$

Supportive measures ${ }^{\#}$

PAH specific therapy

Liver transplantation
HPS

Always present

Sometimes present

Low

Demonstration of intrapulmonary vascular dilatation and increased $\mathrm{A}-\mathrm{a}$ gradient ( $\geqslant 15$ or $20 \mathrm{mmHg}$ )

Arterial blood gas

Contrast enhanced echocardiography and/or lung perfusion scanning

Partial pressure of oxygen in arterial blood: Mild: $\geqslant 80 \mathrm{mmHg}$ Moderate: $\geqslant 60-80 \mathrm{mmHg}$ Severe: $\geqslant 50-60 \mathrm{mmHg}$ Very severe: $<50 \mathrm{mmHg}$

Supportive measures ${ }^{\#}$

Embolisation/coiling of large IPVDs/AVMs Liver transplantation

AVM: arteriovenous malformation; A-a: Alveolar-arterial. \#: supportive measures including supplemental oxygen therapy, diuretics, nutrition and exercise; "': while PoPH may improve post liver transplant, severe PoPH is a contraindication to liver transplantation.

Treatment of PoPH consists of individualised supportive measures and PAH specific therapy. $\beta$-blocker therapy for primary or secondary prophylaxis of variceal bleeding should be prescribed on a case-by-case basis, as they may have detrimental effects on exercise tolerance and pulmonary haemodynamics [2]. Similarly, transjugular intrahepatic portosystemic shunt placement has no role in the management of PoPH and may increase right ventricular preload and precipitate right heart failure [10]. Careful patient selection and preoperative optimisation of $\mathrm{PoPH}$ are associated with a 5-year post liver transplant survival of 78 $81 \%[6,15]$.

However, while PoPH can improve post liver transplantation, severe PoPH often precludes liver transplantation due to the high perioperative mortality $[10,16]$. A mPAP threshold of $>50 \mathrm{mmHg}$ is often considered an absolute contraindication to the same due to the $100 \%$ cardiopulmonary mortality in one meta-analysis [16].

HPS is another important pulmonary vascular complication of portal hypertension. While PoPH is characterised by $\mathrm{PH}$ and increased PVR, isolated HPS is typically associated with intrapulmonary vasodilatation and reduced PVR. However, as PoPH and HPS can occur in the same individual, this can confound the clinical picture somewhat. HPS is classified by a triad of arterial desaturation due to intrapulmonary vasodilatation, platypnoea (dyspnoea which is worse in the upright position) and orthodeoxia (a decrease in partial pressure of oxygen in arterial blood $\left(P_{\mathrm{aO}_{2}}\right)$ of $\geqslant 5 \%$ in the standing position) [9]. Intrapulmonary vasodilatation in HPS may manifest as precapillary and capillary IPVDs, discrete AVMs or a combination of both. These typically occur in the lower lobes and account for worsening of ventilationperfusion mismatch in the standing position, as blood flow is increased to the dependent lower lobes [17].

A diagnosis of HPS requires demonstration of IPVDs and an increased alveolar-arterial oxygen gradient (>15 mmHg) [9]. The severity of disease is subsequently graded by reductions in the $P_{\mathrm{aO}_{2}}$ (table 2) [9]. HPS can be classified as type 1 HPS if there are diffuse IPVDs, and type 2 HPS if the primary pathology are discrete pulmonary AVMs. Relevant investigations include arterial blood gas, contrast enhanced echocardiography and/or lung perfusion scanning with macro-aggregated albumin and occasionally pulmonary angiography [9]. Liver transplantation is potentially curative in this condition and pulmonary vascular abnormalities frequently resolve post-transplant [18].

This case of PoPH in a 43-year-old man with progressive dyspnoea on a background of idiopathic non-cirrhotic portal hypertension and splenectomy highlights important diagnostic and treatment considerations in these complex cases. 


\section{Answer 1}

\section{Correct answers:}

a. Flattening of the intraventricular septum

b. Peak tricuspid regurgitation velocity $>3.4 \mathrm{~m} \cdot \mathrm{s}^{-1}$

d. Right ventricle/left ventricle basal diameter ratio $>1.0$

These echocardiography signs are suggestive of $\mathrm{PH}$ in symptomatic patients [1].

Incorrect answers:

c. Pulmonary artery diameter $>10 \mathrm{~mm}$

e. Peak tricuspid regurgitation velocity $\leqslant 2.8 \mathrm{~m} \cdot \mathrm{s}^{-1}$

A pulmonary artery diameter $>25 \mathrm{~mm}$ can be suggestive of $\mathrm{PH}$ when observed in combination with other echocardiographic features. A peak tricuspid regurgitation velocity $\leqslant 2.8 \mathrm{~m} \cdot \mathrm{s}^{-1}$ indicates a low probability of PH when other signs of $\mathrm{PH}$ are absent.

\section{Answer 2}

\section{Correct answer:}

d. Portopulmonary hypertension (PoPH) is defined by concomitant portal hypertension and pulmonary arterial hypertension (PAH). PoPH is the most likely cause of PH in this case given the patient's history of idiopathic non-cirrhotic portal hypertension since childhood.

Incorrect answers:

a. Hepatopulmonary syndrome is defined by arterial desaturation due to intrapulmonary vascular dilatations (IPVDs), platypnoea (dyspnoea that worse when standing when compared with the supine position) and orthodeoxia (desaturation when moving from the supine to upright position). The PVR is typically low in light of pulmonary vasodilatation. This diagnosis is less likely but requires further investigations including arterial blood gas and contrast enhanced echocardiography to definitively exclude the same.

b. Chronic respiratory failure due to kyphoscoliosis is unlikely to be the primary cause of PH in this case as the patient has normal spirometry and it is described as mild.

c. Chronic thromboembolic pulmonary hypertension has been excluded by normal $V ' / Q^{\prime}$ imaging. Risk factors for this condition in this case include a history of splenectomy.

e. Left heart disease is also unlikely, due to normal left heart size and function on echocardiography and the precapillary pattern of $\mathrm{PH}$ on right heart catheterisation, with an associated PAWP $<15 \mathrm{mmHg}$.

\section{Answer 3}

\section{Correct answer:}

b. The prescription of a $\beta$-blocker for prophylaxis of variceal bleeding in these cases may be associated with deleterious effects on exercise capacity and pulmonary haemodynamics [2].

Incorrect answers:

a. PoPH only occurs in patients with advanced cirrhosis. This is incorrect. PoPH can occur in patients with portal hypertension with or without concomitant liver disease [3]. In this case the patient developed PoPH in isolated portal hypertension and in the absence of concomitant cirrhosis.

c. PoPH is an absolute contraindication to liver transplantation. A diagnosis of PoPH is not an absolute contraindication to liver transplantation. However, pulmonary haemodynamics (including MPAP and PVR) and right ventricular function should be incorporated in the pre-transplant assessment, as right heart dysfunction could result in graft congestion and malfunction [4]. Furthermore, severe PoPH is considered an absolute contraindication to liver transplantation in most centres [5].

d. Endothelin receptor antagonists are contraindicated due to potential liver toxicity. This is incorrect. Endothelin receptor antagonists (ERAs) can be prescribed for PoPH, although the use of phosphodiesterase type 5 inhibitors is more common [6,7]. ERAs were evaluated in adult patients with PoPH and Child-Pugh class A and B cirrhosis in the phase 4 PORTICO study and no hepatic safety concerns were observed [8]. However, drug-induced liver injury is a potentially serious adverse effect and therefore liver function tests should be monitored at periodic intervals.

Patient consent: Verbal and written consent obtained by the patient in this case to publish anonymised patient information.

Conflict of interest: S. Cullivan is the Janssen Pharmaceuticals Newman Fellow in pulmonary hypertension and translational medicine. Janssen Pharmaceuticals had no input in the submitted work and no funding was received for same. B. McCullagh has nothing to disclose. S. Gaine has received honoraria and speaker's fees from Actelion and Janssen Pharmaceuticals, and is an advisory board member for United Therapeutics, outside the submitted work. 


\section{References}

1 Galiè N, Humbert M, Vachiery J-L, et al. 2015 ESC/ERS Guidelines for the diagnosis and treatment of pulmonary hypertension. Eur Respir J 2015; 46: 903-975.

2 Provencher $\mathrm{S}$, Herve $\mathrm{P}$, Jais $\mathrm{X}$, et al. Deleterious effects of beta-blockers on exercise capacity and hemodynamics in patients with portopulmonary hypertension. Gastroenterology 2006; 130: 120-126.

3 Saleemi S. Portopulmonary hypertension. Ann Thorac Med 2010; 5: 5-9.

4 Steadman RH, Ramsay MAE. Chapter 39 - Portopulmonary hypertension and hepatopulmonary syndrome. In: Busuttil RW, Klintmalm GBG, eds. Transplantation of the Liver. 3rd Edn. Philadelphia, W.B. Saunders, 2015 pp. 514-534.

5 Krowka MJ, Fallon MB, Kawut SM, et al. International Liver Transplant Society practice guidelines: diagnosis and management of hepatopulmonary syndrome and portopulmonary hypertension. Transplantation 2016; 100: $1440-1452$.

6 Savale L, Guimas M, Ebstein N, et al. Portopulmonary hypertension in the current era of pulmonary hypertension management. J Hepatol 2020; 73: 130-139.

7 Sithamparanathan S, Nair A, Thirugnanasothy L, et al. Survival in portopulmonary hypertension: outcomes of the United Kingdom National Pulmonary Arterial Hypertension Registry. J Heart Lung Transplant 2017; 36 770-779.

8 Sitbon $\mathrm{O}$, Bosch J, Cottreel E, et al. Macitentan for the treatment of portopulmonary hypertension (PORTICO): a multicentre, randomised, double-blind, placebo-controlled, phase 4 trial. Lancet Respir Med 2019; 7: 594-604.

9 Porres-Aguilar M, Altamirano JT, Torre-Delgadillo A, et al. Portopulmonary hypertension and hepatopulmonary syndrome: a clinician-oriented overview. Eur Respir Rev 2012; 21: 223-233.

10 Savale L, O'Callaghan DS, Magnier R, et al. Current management approaches to portopulmonary hypertension. Int J Clin Pract 2011; 65: 11-18.

11 Thomas C, Glinskii V, de Jesus Perez V, et al. Portopulmonary hypertension: from bench to bedside. Front Med (Lausanne) 2020; 7: 569413.

12 Krowka MJ, Miller DP, Barst RJ, et al. Portopulmonary hypertension: a report from the US-based REVEAL Registry. Chest 2012; 141: 906-915.

13 Simonneau G, Montani D, Celermajer DS, et al. Haemodynamic definitions and updated clinical classification of pulmonary hypertension. Eur Respir J 2019; 53: 1801913.

14 Kmeid M, Liu X, Ballentine S, et al. Idiopathic non-cirrhotic portal hypertension and porto-sinusoidal vascular disease: review of current data. Gastroenterology Res 2021; 14: 49-65.

15 Sadd CJ, Osman F, Li Z, et al. Long-term outcomes and survival in moderate-severe portopulmonary hypertension after liver transplant. Transplantation 2021; 105: 346-353.

16 Krowka MJ, Plevak DJ, Findlay JY, et al. Pulmonary hemodynamics and perioperative cardiopulmonary-related mortality in patients with portopulmonary hypertension undergoing liver transplantation. Liver Transpl 2000; 6: 443-450.

17 Ramsay MAE. Cahpter 40 - Anesthesia for liver transplantation. In: Busuttil RW, Klintmalm GK, eds. Transplantation of the Liver. 2nd Edn. Philadelphia, W.B. Saunders, 2005; pp. 589-606.

18 Arguedas MR, Abrams GA, Krowka MJ, et al. Prospective evaluation of outcomes and predictors of mortality in patients with hepatopulmonary syndrome undergoing liver transplantation. Hepatology 2003; 37: 192-197. 